\title{
Aproximación a la fauna de pseudoescorpiones (Arthropoda: Arachnida) del departamento de Sucre, Caribe colombiano
}

\author{
Approximation to the pseudoscorpion fauna (Arthropoda: Arachnida) of the \\ Department of Sucre, Colombian Caribbean
}

\begin{abstract}
Bedoya-Roqueme Edwin ${ }^{1,2^{\star}}$ Biol; Pérez-Agudelo Madeleissy ${ }^{1}$ Biol; Quirós-Rodriguez Jorge ${ }^{1,2}$ M.Sc.
${ }^{1}$ Universidad de Córdoba, Facultad de Ciencias Básicas, Departamento de Biología. Montería, Colombia. ${ }^{2}$ Universidad de Córdoba, Grupo de Investigación en Biodiversidad Marina y Costera: BIODIMARC.

Carrera 6 No. 76-103, Montería, Colombia.
\end{abstract}

\section{Keywords:}

Taxonomy; pseudoscorpiones; microhabitat; diversity;

Sucre;

Colombia.

\section{Abstract}

In savanna areas and of tropical dry forest of the department of Sucre, Colombian Caribbean, the pseudoescorpion fauna was studied. 38 individuals were collected belonging in four families and six species, being the litter and the bark the most abundant microhabitat. The specie Pseudochthonius heterodentatus Beier, 1946 is reported for the first time from Colombia, their distribution area being increased. The sampling effort allowed to register near $86 \%$ of the pseudoescorpion species, therefore, can be consider a good approximation to the fauna of pseudoescorpion in the department of Sucre.

\section{Palabras Clave:}

Taxonomía; pseudoscorpiones; microhábitat; diversidad;

Sucre;

Colombia.

\section{Resumen}

En áreas sabanizadas y bosque seco tropical del departamento de Sucre, Caribe colombiano, se estudió la fauna de pseudoescorpiones. Se colectaron 38 individuos distribuidos en cuatro familias y seis especies, siendo la hojarasca y la corteza los microhábitats más abundantes. La especie Pseudochthonius heterodentatus Beier, 1946 se reporta por primera vez y se incrementa la distribución conocida hacia Colombia. El esfuerzo de muestreo permitió registrar cerca del $86 \%$ de las especies de pseudoescorpiones, por tanto, se puede considerar que se realizó una buena aproximación de la fauna de pseudoescorpiones en el departamento de Sucre. 


\section{Introducción}

Los pseudoescorpiones son arácnidos relativamente cosmopolitas, su cuerpo y coloración se encuentra adaptados a los microhábitat que ocupan, comúnmente pueden ser encontrados habitando la hojarasca, debajo de piedras y rocas, debajo de corteza de árboles y troncos en descomposición o en el medio subterráneo y en ecosistemas de ambientes marinos (HOFF, 1959; GABBUTT, 1962; WEYGOLDT, 1969; LEE 1979; MAHNERT y SCHUSTER, 1981; HARVEY et al., 2007; DEL-CLARO y TIZO-PEDROSO, 2009; HARVEY, 2009; 2013; MAHNERT, 2014; BEDOYA-ROQUEME, 2015; JUDSON, 2016).

La fauna de pseudoescorpiones de Colombia, actualmente se encuentra representada por 35 especies (CEBALLOS y FLÓREZ, 2007; MURIENNE et al., 2008; BEDOYA-ROQUEME et al., 2014; 2015; 2016; QUIRÓSRODRÍGUEZ et al., 2015; BEDOYA-ROQUEME, 2015; ROMERO-ORTIZ, 2015); datos subvalorados si se tiene en cuenta los altos valores de diversidad presentes en el país y su influencia tropical (CEBALLOS y FLÓREZ, 2007; MURIENNE et al., 2008; BEDOYA-ROQUEME et al., 2014; 2015; 2016; QUIRÓS-RODRÍGUEZ et al., 2015; BEDOYA-ROQUEME, 2015; ROMERO-ORTIZ, 2015). En la región Caribe colombiana, son escasas las investigaciones realizadas sobre la fauna de pseudoescorpiones y el conocimiento que se tiene es insatisfactorio. Los primeros reportes fueron realizados hace más de un siglo por BANKS (1909) quien describió la especie Garypus viridans de Santa Marta (Magdalena). Posteriormente, MUCHMORE (1982; 1993) describió las especies Ideoblothrus colombiae e I. kochalkai en Santa Marta y Dasychernes inquilinus para las localidades de Rio Frio y Sevilla (Magdalena). En la actualidad, sólo se conoce el reporte de Parachernes melanopygus Beier, 1959, en zonas de bosque seco $\mathrm{y}$, además, los reportes de áreas de manglar para el departamento de Córdoba (BEDOYA-ROQUEME et al., 2014; 2016; BEDOYA-ROQUEME, 2015). En el departamento de Sucre, no se habían realizado reportes sobre estos arácnidos y al no contar con investigaciones realizadas, la principal problemática radica en el desconocimiento de aspectos taxonómicos y bioecológicos, como su composición, riqueza y distribución; por tal motivo, se consideró conveniente iniciar estudios sobre la fauna de pseudoescorpiones, que permitan conocer la diversidad taxonómica en esta región, siendo objeto de estudio la presente aproximación.

\section{Materiales y métodos}

El departamento de Sucre se encuentra ubicado en el noroeste de Colombia entre los $10^{\circ} 08^{\prime} 03^{\prime \prime}$ y $0,8^{\circ} 16^{\prime} 46^{\prime \prime}$ $\mathrm{N}$ y los $74^{\circ} 32^{\prime} 53^{\prime \prime}$ y $75^{\circ} 42^{\prime} 25^{\prime \prime} \mathrm{W}$, dividido en cinco subregiones: Morrosquillo, Sabanas, Montes de María, San Jorge y La Mojana, que constituyen áreas de sabanas, bosque seco tropical (Bs-T) que hace parte del cinturón árido pericaribeño y con un paisaje característico de montaña, con temperatura promedio anual de $28^{\circ} \mathrm{C}$, pluviosidad promedio de 1.000-1.200 $\mathrm{mm}$ anuales; régimen de lluvia bimodal, al corto período de lluvias del primer semestre le sigue un breve período seco entre los meses de junio y julio; en el segundo semestre se presenta la mayor cantidad de precipitación pluvial, (HERNÁNDEZ y SÁNCHEZ, 1992; DÍAZ et al., 2009; GÓMEZ et al., 2013; ANGARITA et al., 2014; SAMPEDRO et al., 2014; PÉREZ et al., 2016).

La recolección de pseudoescorpiones se realizó durante el mes de abril de 2013, para lo cual, se definieron cuatro muestreos por semana en diferentes localidades del departamento de Sucre (Fig. 1), posicionados geográficamente con la ayuda de un GPS marca Garmin ( $\pm 15 \mathrm{~m}$ ), que constituyen áreas sabanizadas y de bosque seco (Tabla 1). La selección obedece a su estado de conservación, debido a que esta zona se encuentra fuertemente influenciada por el uso del suelo, dirigida hacia las prácticas agrícolas y ganaderas que han ocasionado la reducción, fragmentación y deterioro de los bosques (ANGARITA et al., 2014).

En cada localidad se estableció un punto de muestreo con un área georreferenciada aproximada de $100 \mathrm{~m}^{2}$, la selección obedece a características relacionadas con su estado de conservación, la estructura vegetal y fácil acceso. Se adaptaron y se aplicaron las metodologías propuestas por GABBUTT (1970), MAHNERT y ADÍS (2002) y se siguieron las recomendaciones realizadas por ADIS et al. (1988). Para la colecta de la hojarasca se utilizó un cuadrante delimitando un área de $1 \mathrm{~m}^{2}$ con tres repeticiones al azar, con una duración de 12 horas, para un total de 12 unidades muestreables (MAHNERT y ADÍS, 2002); la extracción de la fauna de pseudoescorpiones se realizó a través del procesamiento por embudo de Berlese de la hojarasca colectada (GABBUTT, 1970); de igual forma, teniendo en cuenta los microhábitat que ofrecieran el potencial de encontrarlos, se empleó la búsqueda libre sin restricción de los ejemplares en la corteza de árboles, debajo de piedras, troncos en descomposición, en hojarasca y suelo (MAHNERT y ADIS, 2002).

Los ejemplares inicialmente fueron depositados en viales, conservados en alcohol $70 \%$, almacenados en frascos de boca ancha y etiquetados con información que incluía código de la muestra, sector y fecha para su posterior exanimación en laboratorio (CHAMBERLIN, 1931; HARVEY, 1992; MAHNERT y ADIS, 2002). Se realizaron montajes no permanentes en glicerina para el estudio de los ejemplares con la ayuda de un microscopio Carl Zeiss, plus Axiostar, Alemania 
(CHAMBERLIN, 1931; HARVEY, 1992; HARVEY et al., 2012), proceso que se llevó a cabo en el laboratorio de Microscopia de la Universidad de Córdoba. Los ejemplares fueron sometidos a procesos de aclaramientos con ácido láctico $70 \%$ por difusión lenta de las partes diseccionadas (una pata I y otra IV, un quelícero y un pedipalpo, del cual ha sido separada la pinza) (JUDSON, 1992).

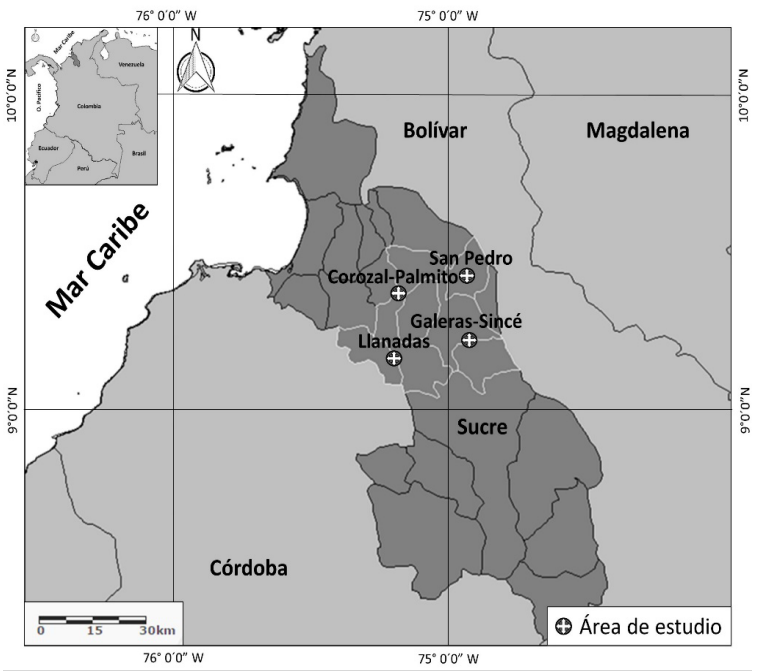

Figura 1. Área de estudio y localización de cada una de las estaciones de muestreo, departamento de Sucre, Caribe colombiano.

Tabla 1. Ubicación de las estaciones de muestreo en el departamento de Córdoba.

\begin{tabular}{cccc}
\hline Localidades & Latitud norte $(\mathbf{N})$ & Longitud oeste $(\mathbf{W})$ & Sectores \\
\hline Las Llanadas & 9.154572 & -75.280523 & Las Llanadas \\
Galeras-Sincé & 9.200737 & -75.072099 & Galeras \\
Corozal-Palmito & 9.358900 & -75.281694 & Corozal \\
San Pedro & 9.403488 & -75.031875 & San Pedro \\
\hline
\end{tabular}

Posteriormente, los ejemplares se depositaron en un microvial y conservados en alcohol $70 \%$ en el mismo vial que el resto del animal. Para la identificación de los ejemplares se utilizaron las claves taxonómicas para familia y género de HARVEY (1992), MAHNERT y ADIS (2002) y BUDDLE (2010), para la identificación de especies y/o especies a fin, se revisaron las descripciones realizadas por HOFF (1945, 1946); VACHON (1951); BEIER (1931, 1932, 1954); HOFF (1964); MUCHMORE (1975); MANHERT (1979); CALLAINI (1986); MANHERT (2001); MAHNERT et al. (2011); KLAUSSEN (2005); TOOREN (2002; 2011). Finalmente, fueron depositados en la colección de pseudoescorpiones del Laboratorio de Entomología de la Universidad de Córdoba (LEUC) con códigos Ps-010, Ps-011, Ps-012, Ps-013, Ps-014, Ps-015, Ps-016, Ps-017, Ps-018, Ps-019, Ps-020. Adicionalmente, la riqueza esperada fue estimada con los estimadores no paramétricos Bootstrap, Jackknife 1 y Jackknife 2, utilizando EstimateS 9.1 (COLWELL, 2013).

\section{Resultados y discusión}

Se colectaron 38 individuos pertenecientes a cuatro familias y seis especies (Fig. 2), Chernetidae y Olpiidae presentaron el mayor número de especies (2), seguida de Atemnidae y Chthoniidae con una especie (Tabla 2). Pachyolpium aff. granulatum Beier, 1954, y Apolpium sp. (Olpiidae) fueron las especies más abundantes (47.3\%), seguida de Paratemnoides nidificator (Balzan, 1888) (Atemnidae) (34.2\%) y Pseudochthonius heterodentatus Hoff, 1946, (Chthoniidae), Americhernes sp. y Pseudopilanus sp., que son las menos abundantes. (7.89\%) (Tabla 2).

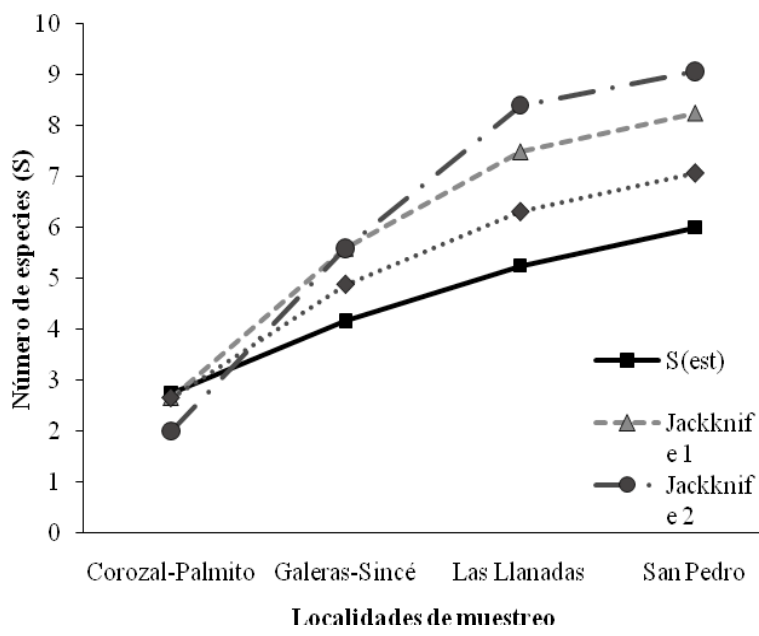

Figura 2. Curva de acumulación de especies pseudoescorpiones del departamento de Sucre. Sest: riqueza estimada. Estimadores paramétricos: Bootstrap, Jackknife 1 y Jackknife 2.

Tabla 2. Pseudoescorpiones colectados indicando familia y especie.

\begin{tabular}{|c|c|c|c|c|}
\hline \multirow[b]{2}{*}{ Familia / Especie } & \multicolumn{4}{|c|}{ Estaciones de muestro } \\
\hline & $\begin{array}{l}\text { Corozal- } \\
\text { Palmito }\end{array}$ & $\begin{array}{l}\text { Galeras- } \\
\text { Sincé }\end{array}$ & $\begin{array}{c}\text { Las } \\
\text { Llanadas }\end{array}$ & $\begin{array}{c}\text { San } \\
\text { Pedro }\end{array}$ \\
\hline \multicolumn{5}{|l|}{ Atemnidae } \\
\hline $\begin{array}{l}\text { Paratemnoides nidificator } \\
\text { (Balzan, 1888) }\end{array}$ & 0 & 1 & 0 & 9 \\
\hline \multicolumn{5}{|l|}{ Chernetidae } \\
\hline Americhernes sp. & 0 & 0 & 0 & 3 \\
\hline Pseudopilanus sp. & 0 & 3 & 0 & 0 \\
\hline \multicolumn{5}{|l|}{ Olpiidae } \\
\hline $\begin{array}{l}\text { Pachyolpium aff. granulatum } \\
\text { Beier, } 1954\end{array}$ & 7 & 4 & 2 & 1 \\
\hline Apolpium sp. & 0 & 2 & 3 & 0 \\
\hline $\begin{array}{l}\text { Chthoniidae } \\
\text { Pseudochthonius heterodentatus } \\
\text { Hoff, } 1946\end{array}$ & 0 & 3 & 0 & 0 \\
\hline Total & 7 & 13 & 5 & 13 \\
\hline
\end{tabular}

* Nuevo reporte para Colombia.

El microhábitat que presentó el mayor número de individuos fue la hojarasca 23 individuos $(60 \%)$, seguido de corteza con 12 individuos $(31,5 \%)$ y suelo con tres individuos $(7,8 \%)$; las especies $P$. aff. granulatum, 
Apolpium sp.; mientras que $P$. nidificatory Americhernes sp., se encontraron asociados a corteza de árboles; $P$. heterodentatus se encontró en suelo y Pseudopilanus sp., en troncos en descomposición.

\section{Taxonomía.}

\section{Familia Chthoniidae}

Pseudochthonius heterodentatus. Fig. 3A

Material examinado: $2 \hat{\jmath}, 1$, Galeras, departamento de Sucre, Colombia: Galeras, $9^{\circ} .200737$ N, $-75^{\circ} .072099$ W, abril de 2013, áreas de sabana, E., BedoyaRoqueme; J., Jiménez. Leg (depositados en LEUC; Ps-020).

Distribución: Brasil, Trinidad y Tobago, Colombia.

Observación: la especie $P$. heterodentatus es conocida sólo de las localidades de Trinidad y Tobago y los reportes de Brasil (HOFF, 1946; HARVEY, 2013). Los ejemplares colectados en Galeras-Sucre (Colombia) pertenecen a esta especie, sin embargo, las diferencias en cuanto a longitud corporal: $\hat{\jmath}, 1,43-1,45$ $(q, 1,6)$; longitud de la pinza: $\hat{\jmath}, 0,658-0,67 / 0,105-0,11$ $(+, 0,83 / 0,14)$; longitud de la mano: $\hat{\jmath}, 0,19-0,2 / 0,11-$

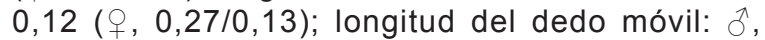
0,458-0,5/0,05-0,06 ( $+, 0,53)$; pero estas pequeñas diferencias probablemente pueden atribuirse a la variación geográfica de las especies, debido a que todos los ejemplares presentan más o menos algunas diferencias.

\section{Familia Olpiidae \\ Apolpium sp. Fig. 3B}

Material examinado: 2 , Galeras, departamento de Sucre, Colombia: Galeras, $9^{\circ} .200737$ N, $-75^{\circ} .072099$ W, abril de 2013, áreas de sabana, E., Bedoya-Roqueme;

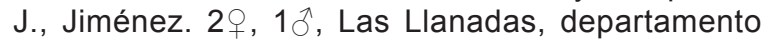
de Sucre, Colombia: Las Llanadas, 9․154572 N, $-75^{\circ} .280523 \mathrm{~W}$, abril de 2013, áreas de sabana, E., Bedoya-Roqueme; J., Jiménez. Leg (depositados en LEUC; Ps-018, Ps-019).

Distribución: Brasil, Colombia, Costa Rica, Ecuador, Trinidad y Tobago, Venezuela.

Pachyolpium aff. granulatum. Fig. 3C

Material examinado: 5 , 3 , Corozal, departamento de Sucre, Colombia: Corozal-Palmito: $9^{\circ} .358900 \mathrm{~N}$, $-75^{\circ} .281694 \mathrm{~W}$, áreas de sabana, E., Bedoya-Roqueme;

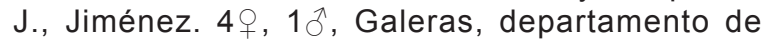
Sucre, Colombia: Galeras, $9^{\circ} .200737$ N, $-75^{\circ} .072099$ W, abril de 2013, Zonas de pastizales, E., BedoyaRoqueme; J., Jiménez. 2 Tritoninfas, Las Llanadas, departamento de Sucre, Colombia: Las Llanadas, $9^{\circ} .154572 \mathrm{~N},-75^{\circ} .280523 \mathrm{~W}$, abril de 2013 , áreas de sabana, E., Bedoya-Roqueme; J., Jiménez. $1 \hat{\delta}$, San Pedro, departamento de Sucre, Colombia: Las Llanadas, $9^{\circ} .403488 \mathrm{~N},-75^{\circ} .031875 \mathrm{~W}$, abril de 2013 ,
Zonas de Cultivos, E., Bedoya-Roqueme; J., Jiménez. Leg (depositados en LEUC; Ps-014, Ps-015, Ps-016, Ps-017).

Distribución: Colombia, Perú, Surinam, Venezuela.

Observación: De acuerdo a la descripción realizada por BEIER (1954) y la clave suministrada por TOOREN (2011) los ejemplares comparten con la especie Pachyolpium granulatum el nivel de posición del tricobotrio est respecto a los tricobotrios it e ist sobre el dedo fijo de la pinza del pedipalpo; la longitud y anchura de la patela del pedipalpo $(\hat{0}, 0,60-0,62 / 0,25-$ $0,27 \mathrm{~mm}$; + , 0,71-0,73/0,30-0,31 mm) y del fémur (ठ, 0,61-0,63/0,20-0,23 mm; ᄋ, 0,69-0,71/0,23$0,27 \mathrm{~mm}$ ); el margen interno del fémur ligeramente cóncavo y el aspecto de la gálea con tres ramas terminales; sin embargo, esto ejemplares de SucreColombia, difieren de la descripción de $P$. granulatum de Venezuela (BEIER, 1954) porque presentan una irregular y asimétrica quetotaxia de los terguitos: (particularmente en los terguitos III y VII) $\hat{\partial}$, terguitos I-XII: 6:6:6:8:10:10:10:12:12:12(2ST):10(2ST):4(2ST); q, terguitos I-XII: 6:6:8:8:8:10:10:12:12:10(2ST):10(2 $\mathrm{ST}): 4$ (2ST), mientras que en $P$. granulatum presenta una quetotaxia como sigue: $\hat{\delta}$, + , 6:6:8:8:10:10:12:12:12, por esta razón son separados de la descripción tipo de $P$. granulatum Beier, 1954, sin presentar otras diferencias morfológicas evidentes.

\section{Familia Atemnidae}

Paratemnoides nidificator. Fig. 3D

Material examinado: $1 \hat{\delta}$, Galeras, departamento de Sucre, Colombia: Galeras, $9^{\circ} .200737$ N, $-75^{\circ} .072099$ W, abril de 2013, Zonas de pastizales, E., BedoyaRoqueme; J., Jiménez. 6ㅇ, 3 $\hat{\jmath}$, San Pedro, departamento de Sucre, Colombia: Las Llanadas, $9^{\circ} .403488 \mathrm{~N}$, $-75^{\circ} .031875$ W, abril de 2013, Zonas de Cultivos, E., Bedoya-Roqueme; J., Jiménez. Leg (depositados en LEUC; Ps-010, Ps-011).

Distribución: Argentina, Brasil, Colombia, Costa Rica, Ecuador, Guatemala, Guyana, Haití, México, Panamá, Paraguay, Perú, San Vicente y las granadinas, Venezuela.

\section{Familia Chernetidae}

Americhernes sp. Fig. 3F

Material examinado: $3 \propto$, San Pedro, departamento de Sucre, Colombia: Las Llanadas, $9^{\circ} .403488 \mathrm{~N}$, $-75^{\circ} .031875$ W, abril de 2013, Zonas de Cultivos, E., Bedoya-Roqueme; J., Jiménez. Leg (depositados en LEUC; Ps-012).

Distribución: Argentina, Australia, Brasil, Belice, Canadá, Chile, Cuba, Reino Unido, México, Polinesia Francesa, Puerto Rico, Paraguay, Estados Unidos, Samoa. 


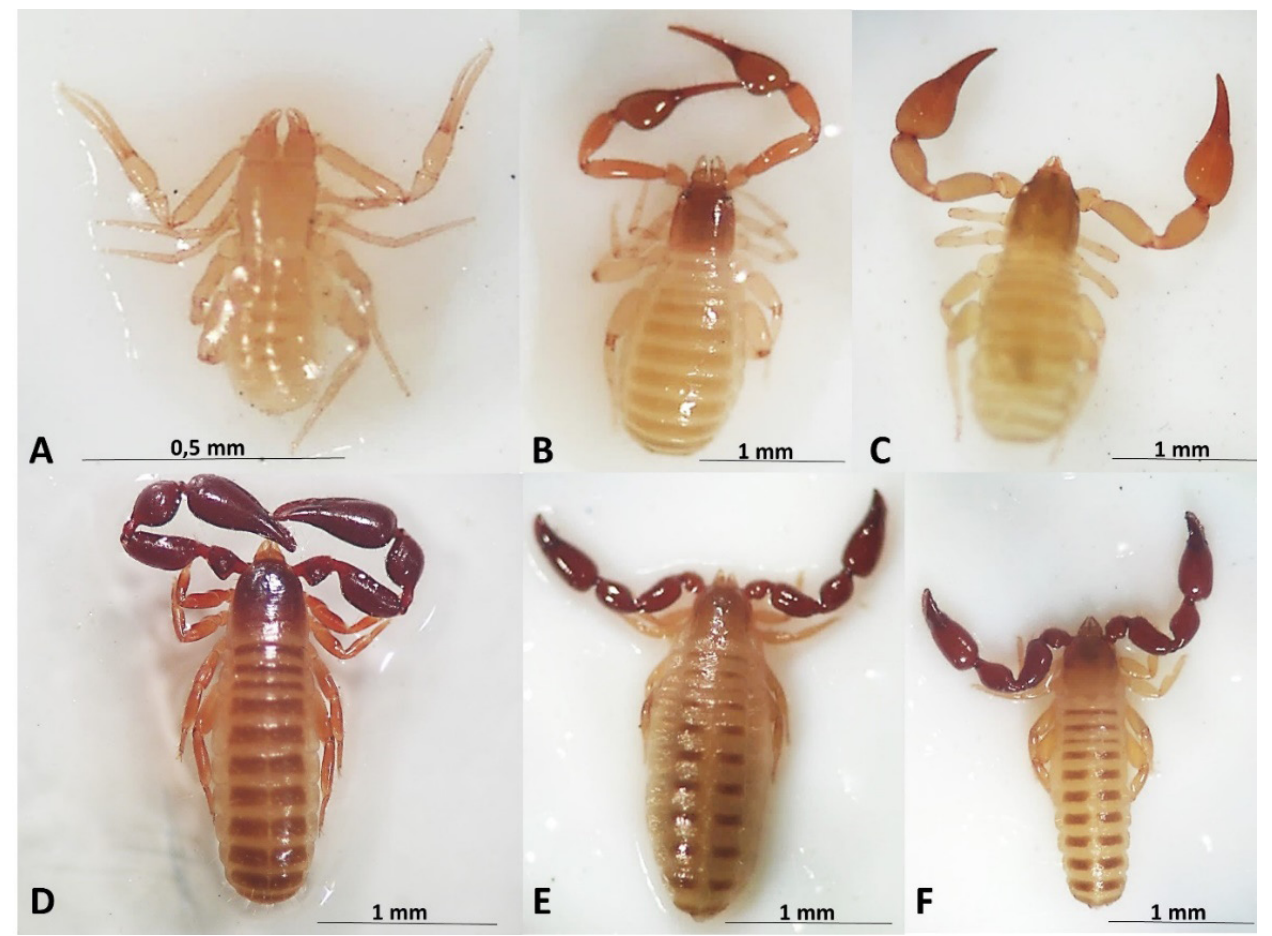

Figura 3. Pseudoescorpiones del departamento de Sucre, Habitus: A, Pseudochthonius heterodentatus. B, Apolpium sp. C, Pachyolpium aff. granulatum. D, Paratemnoides nidificator. E, Pseudopilanus sp., $\mathrm{F}$, Americhernes sp.

Pseudopilanus sp. Fig. 3E

Material examinado: $2 \hat{\delta}, 1+$ Galeras, departamento de Sucre, Colombia: Galeras, $9^{\circ} .200737 \mathrm{~N},-75^{\circ} .072099 \mathrm{~W}$, abril de 2013, corteza de árboles en descomposición, E., Bedoya-Roqueme; J., Jiménez. Leg (depositados en LEUC; Ps-013).

Distribución: Argentina, Brasil, Chile, Ecuador, Paraguay.

El departamento de Sucre se caracteriza por estar dividido en cinco subregiones, El esfuerzo de muestreo realizado en las cuatro localidades del departamento de Sucre, permitió registrar cerca del $86 \%$ de las especies de pseudoescorpiones, la curva de acumulación de especies se acercó a una tendencia asintótica, alcanzando un valor máximo de diez especies para las localidades de acuerdo con los estimadores Jackknife 1 y Jackknife 2 (Fig. 2). Por tanto, con base en estos resultados, el valor de riqueza observada en las cuatro localidades (seis especies) se acerca al valor esperado, lo cual indica una buena representatividad del muestreo en las cuatro localidades.

Morrosquillo, Sabanas, Montes de María, San Jorge y La Mojana y constituyen áreas de sabanas, bosque seco tropical (Bs-T) (HERNÁNDEZ y SÁNCHEZ, 1992; DÍAZ et al., 2009; GÓMEZ et al., 2013; ANGARITA et al., 2014; SAMPEDRO et al., 2014; PÉREZ et al., 2016). Los pseudoescorpiones son arácnidos que aparecen asociados a muchos microhábitats como guano de murciélago, nidos de hormigas y de abejas, y partes de plantas; de igual forma, se les conoce comportamientos foréticos en aves, insectos y mamíferos (WEYGOLDT, 1969; DEL-CLARO y TIZO-PEDROSO, 2009).

Constituyen un grupo mesodiverso de arácnidos (HARVEY, 2013); sin embargo, solo seis especies fueron registradas presentando una baja riqueza para las localidades muestreadas en este estudio, algunos autores como WEYGOLDT (1969); GABBUTT (1970); MORÁIS y ADIS (1997); AGUIAR et al. (2006) sugieren una estrecha relación de la abundancia de los pseudoescorpiones con la estructura vegetal de la zona; asimismo, estos arácnidos se encuentran ocupando uno o varios microhábitats (MURIENNE et al., 2008; BATTIROLA et al., 2017).

Los altos valores de abundancia en la hojarasca, sugiere que este microhábitat donde se encuentran establecidos, ofrece, todas las condiciones necesarias tanto de refugio como de alimento (WEYGOLDT, 1969; MORAIS y ADIS, 1997). La mayoría de las especies de pseudoescorpiones son terrícolas y su presencia en corteza se asocia a fenómenos de migración o 
sitios de anidación (WEYGOLDT, 1969; BATTIROLA et al., 2017). Asimismo, la baja frecuencia en el suelo se puede asociar con adaptaciones a la vida de estos entornos sometidos a variaciones en las condiciones de la estructura vegetal (MORAIS y ADIS, 1997; BATTIROLLA et al., 2017), presentes en áreas de sabana y zonas de cultivo.

\section{Conclusión}

A pesar de los pocos individuos colectados el presente estudio, se convierte en el primer reporte de pseudoescorpiones en el departamento de Sucre. Se reporta por primera vez y se incrementa la distribución conocida de la especie Pseudochthonius heterodentatus Beier, 1946 hacia Colombia; de igual forma, se reporta por primera vez para el departamento de Sucre seis especies. Por tanto, se puede considerar que se realizó una buena aproximación de la fauna de pseudoescorpiones en el departamento de Sucre, sin embargo, quedan ciertas zonas por explorar y es necesario realizar nuevos estudios enfocados aplicando y combinando diferentes metodologías para obtener mayores valores de riqueza de la fauna de pseudoescorpiones del departamento de Sucre.

Agradecimientos: Expresamos nuestro más sincero agradecimiento a la Dra. Doris Helena Serrano y al grupo de investigación Geografía y Medio Ambiente, por facilitar los muestreos, enmarcados en el proyecto "Efectos y cambios en la cobertura y uso del suelo de Las Sabanas de los departamentos de Córdoba, Sucre y la zona semidesértica de La Guajira, mediante la utilización de imágenes de satélite" CIUC-Universidad de Córdoba. De igual forma, a Jheysson Jiménez Sáez por su colaboración durante la investigación en la colecta del material de estudio.

\section{Referencias}

ADIS, J., MAHNERT, V.; MORÁIS, J. W.; RODRÍGUEZ, J.M. 1988. Adaptation of an Amazoniaian pseudoscorpion (Arachnida) from dryland forests to inundation forests. Ecology 69 (1):287-291.

AGUIAR, N.O.; GUALBERTO, T.; FRANKLIN, E. 2006. A mediumspatial scale distribution pattern of Pseudoscorpionida (Arachnida) in a gradient of topography (altitude and inclination), soil factors, and litter in a central Amazonia forest reserve, Brazil. Brazilian Journal of Biology 66:791-802.

ANGARITA, H.D.; SANMARTÍN, S.D.; MERCADO, G.J. 2014. Nuevos registros Corológicos para Sucre (Sanguaré - Colombia). Revista Colombiana de Ciencia Animal 6 (2):360-373.

BANKS, N. 1909. New tropical pseudoscorpions. Journal of the New York Entomological Society 17:145-148.

BATTIROLA, L.D.; ROSADO-NIETO, G.H.; BATISTELLA, D.A.; MAHNERT, V.; BRESCOVIT, A.D.; MARQUES, M.I. 2017. Vertical and time distribution of Pseudoscorpiones (Arthropoda: Arachnida) in a floodplain forest in the Brazilian Pantanal. Revista Biología Tropical 65 (2):445-459.

BEDOYA-ROQUEME, E. 2015. Una nueva especie de Paraliochthonius (Pseudoscorpiones: Chthoniidae: Tyrannochthoniini) de Colombia. Revista Ibérica de Aracnología 26:49-54.

BEDOYA-ROQUEME, E.; BEDOYA, R.; QUIROS, J.A. 2015. Primer reporte del género Cryptocheiridium (Pseudoscorpiones: Cheiridiidae: Cheiridiinae) en Colombia. Revista Colombiana de Ciencia Animal 7 (1):11-18.

BEDOYA-ROQUEME, E.; SALLEG, P.; QUIROS, A. 2014. Primer reporte de pseudoescorpiones (Arachnida: Pseudoscorpionida) en el departamento de Córdoba, Caribe colombiano. Revista Colombiana Ciencia Animal 6 (1):177-183.

BEDOYA-ROQUEME, E.; SALLEG, P.G.; QUIRÓS, J.A. 2016. Sobre la ecología de pseudoescorpiones (Arachnida: Pseudoscorpiones) en bosques de manglar del sur del golfo de Morrosquillo, Caribe Colombiano. Revista Ibérica de Aracnología 28:65-74.

BEIER, M. 1931. Zur Kenntnis der Chthoniiden (Pseudoskorpione). Zoologischer Anzeiger 93:49-56.

BEIER, M. 1932. Pseudoscorpionidea I. Subord. Chthoniinea et Neobisiinea. Tierreich 57:1-258. 
BEIER, M. 1954. Eine Pseudoscorpioniden-Ausbeute aus Venezuela. Memorie del Museo Civico di Storia Naturale di Verona 4:131-142.

BUDDLE, C. 2010. Photographic key to the Pseudoscorpions of Canada and the adjacent USA. Canadian Journal of Arthropod Identification 10:1-77.

CALLAINI, G. 1986. Appunti su alcune specie italiane della famiglia Chernetidae Menge (Arachnida, Pseudoscorpionida). Notulae Chernetologicae XV. Bollettino del Museo Civico di Storia Naturale Verona 11:379-401.

CEBALLOS, A.; FLOREZ, E. 2007. Pseudoescorpiones de Colombia (Arachnida: Pseudoscorpiones): lista actualizada de especies. Biota Colombiana 8 (1): 47-51.

CHAMBERLIN, J.C. 1931. The arachnid order Chelonethida. Stanford University Publications, Biological Sciences $7(1): 1-284$.

COLWELL, R. 2013. EstimateS: Statistical estimation of species richness and shared species from samples Version 9.1. User's Guide and application. Disponible en: URL: http://www.viceroy.eeb.uconn.edu/estimates. Consultado 17-05-2017.

DEL-CLARO, K.; TIZO-PEDROSO, E. 2009. Ecological and evolutionary pathways of social behavior in Pseudoscorpions (Arachnida: Pseudoscorpiones). Acta Ethologica 12:13-22.

DÍAZ, P.; JORGE A.; MOLANO, P.; CARLOS E.; GAVIRIA, B.; JULIO C. 2009. Diversidad genérica de hormigas (Hymenoptera: Formicidae) en ambientes de Bosque Seco de Los Montes de María, Sucre, Colombia. Revista Colombiana de Ciencia Animal 1 (2):279-285.

GABBUTT, P.D. 1962. Nets of the marine falsescorpion. Nature London 196: 97-98.

GABBUTT, P.D. 1970. Sampling problems and the validity of life history analyses of pseudoscorpions. Journal of Natural History 4:1-15.

GÓMEZ, A.D.; MARTINEZ, A.J.; MENDOZA, P.H.; ALVAREZ, G.D.; RUIZ, P.S. 2013. Registro de escorpiones (Chelicerata: Scorpiones) para el departamento de Sucre, Colombia. Revista Colombiana Ciencia Animal 5 (1):150153.

HARVEY, M.S. 1992. The phylogeny and classification of the Pseudoscorpionida (Chelicerata: Arachnida). Invertebrate Taxonomy 6:1373-1435.

HARVEY, M.S. 2009. The first Australasian species of the genus pseudoscorpion halophilic Paraliochthonius (Pseudoscorpiones: Chthoniidae). Records of the Western Australian Museum 25:329-344.

HARVEY, M.S. 2013. Pseudoscorpions of the World, version 2.0. Western Museum Australian. Disponible en: URL: Http:www.museum.wa.gov.au /catalogs/pseudoscorpion. Consultado: 20-01-2017

HARVEY, M.S.; RATNAWEERA, P.B.; UDAGAMA, P.V.; WIJESINGHE, M.R. 2012. A new species of the pseudoscorpion genus Megachernes (Pseudoscorpiones: Chernetidae) associated with a threatened Sri Lankan rainforest rodent, with a review of host associations of Megachernes. Journal of Natural history 46:2519-2535.

HARVEY, M.S.; WALDOCK, J.; TEALE, R.; WEBBER, J. 2007. New distribution records of the intertidal pseudoscorpion Parahya submersa (Pseudoscorpiones: Parahyidae). Records of the Western Australian Museum 23:393-395.

HERNÁNDEZ, C.J.; SÁNCHEZ, E. 1992. Biomas terrestres de Colombia. Págs. 105-151 en: Halffter, G (ed.), La diversidad biológica de Iberoamérica I. CYTED. Inst. Mex. de Ecología y Secretaría de Desarrollo Social. México. HOFF, C.C. 1945. The pseudoscorpion subfamily Olpiinae. American Museum Novitates 1291:1-30.

HOFF, C.C. 1946. A redescription of Atemnus elongatus Banks, 1895. Proceedings of the New England Zoölogical Club 23:109-113. 
HOFF, C.C. 1946. Three new species of heterosphyronid pseudoscorpions from Trinidad. American Museum Novitates 1322:1-13.

HOFF, C.C. 1959. The ecology and distribution of the pseudoscorpions of north-central New Mexico. University New Mexico Publishing Biological 8:1-68.

HOFF, C.C. 1964. The pseudoscorpions of Jamaica. Part 3. The suborder Diplosphyronida. Bulletin of the Institute of Jamaica, Science Series 10 (3):1-47.

JUDSON, M.L.I. 1992. A simple, Slow-diffusion Method for clearing small arthropods. Newsl. Br. Arachnologica Society 64: 6-7.

JUDSON, M.L.I. 2016. Pseudoscorpions (Arachnida, Chelonethi) in Mexican amber, with a list of extant species associated with mangrove and Hymenaea trees in Chiapas. Boletín de la Sociedad Geológica Mexicana 68 (1):57-79.

KLAUSEN, F.E. 2005. The male genitalia of the family Atemnidae (Pseudoscorpiones). Journal of Arachnology 33:641-662.

LEE, V.F. 1979. The maritime pseudoscorpions of Baja California, México (Arachnida: Pseudoscorpionida). Occasional Papers of the California Academy of Sciences 131 (i-iv):1-38.

MAHNERT, V. 1979. Pseudoskorpione (Arachnida) aus dem Amazonas-Gebiet (Brasilien). Revue Suisse de Zoologie 86:719-810.

MAHNERT, V. 2001. Cave-dwelling pseudoscorpions (Arachnida, Pseudoscorpiones) from Brazil. Revue Suisse de Zoologie 108:95-148.

MAHNERT, V. 2014. Pseudoscorpions (Arachnida: Pseudoscorpiones) from the Galapagos Islands (Ecuador), Revue Suisse de Zoologie 121 (2):135-210.

MAHNERT, V.; ADIS, J. 2002. Pseudoscorpiones. Págs. 367-380 en: Adis, J. (ed.), Amazonian Arachnida and Myriapoda. Pensoft Publisher. Sofía, Moscow.

MAHNERT, V.; DI IORIO, O.; TURIENZO, P.; PORTA, A. 2011. Pseudoscorpions (Arachnida) from Argentina. New records of distributions and habitats, corrections and an identification key. Zootaxa 2881:1-30.

MAHNERT, V.; SCHUSTER, R.1981. Pachyolpium atlanticum sp. nov., ein Pseudoskorpion aus der Gezeitenzone der Bermudas-Morphologie und Ökologie (Pseudoscorpiones: Olpiidae). Revue Suisse de Zoologie 88:265-273.

MORÁIS, J.; ADIS, J. 1997. Abundance and phenology of Pseudoscorpiones (Arachnida) from a mixedwater inundation forest in Central Amazonia, Brazil. Revue Suisse de Zoologie 104:475-483.

MUCHMORE, W.B. 1975. Use of the spermathecae in the taxonomy of chernetid pseudoscorpions. Págs. 17-20 en: Universidad de Amsterdam (ed.), Proceedings of the 6th International Arachnological Congress. Universidad de Amsterdam, Amsterdam.

MUCHMORE, W.B. 1982. The genera Ideobisium and Ideoblothrus, with remarks on the family Syarinidae (Pseudoscorpionida). Journal of Arachnology 10: 193-221.

MUCHMORE, W.B. 1993. Annotated list and bibliography of Pseudoscorpionida (Arachnida) reported from the Caribbean region. I. Trinidad, Venezuela and Colombia, and including Aruba, Bonaire, and Curaçao. Tropical Zoology Special Issue 1:83-104.

MURIENNE, J.; HARVEY, M.S.; GIRIBET, G. 2008. First molecular phylogeny of the major clades of Pseudoscorpiones (Arthropoda: Chelicerata). Molecular Phylogenetics and Evolution 49:170-184. 
MURIENNE, J.; HARVEY, M.S.; GIRIBET, G. 2008. First molecular phylogeny of the major clades of Pseudoscorpiones (Arthropoda: Chelicerata). Molecular Phylogenetics and Evolution 49:170-184.

PÉREZ, C.C.; PEROZA-CORONADO, V.; PÉREZ-CORDERO, A. 2016. Colonización de hongos formadores de micorrizas arbusculares en raíces de pasto colosoana y angletón en cinco localidades del departamento de Sucre. Revista Colombiana de Ciencia 8 (2):214-218.

QUIRÓS-RODRÍGUEZ, J.A.; BEDOYA-ROQUEME, E.; BEDOYA, R. 2015. Primer reporte de la familia Cheiridiidae (Arachnida: Pseudoscorpionida) en Colombia. Acta Biológica Colombiana 20 (3): 217-220.

ROMERO-ORTIZ, C. 2015. Orden Pseudoscorpiones (Chelonethi). Págs. 89-108 en: Flórez, E.; Romero-O, C.; López, D.S. (ed.), Los artrópodos de la reserva natural río Ñambí. Instituto de Ciencias Naturales, Universidad Nacional de Colombia, Bogotá D.C.

SAMPEDRO, M.A.; GÓMEZ, F.H; BALLUT, D.G. 2014. Estado de la vegetación en localidades abandonadas por "desplazamiento", en los Montes de María Sucre, Colombia. Revista Colombiana de Ciencia Animal 6 (1): $184-193$.

TOOREN, D. 2002. Pseudoscorpions of the genera Pachyolpium, Novohorus and Amblyolpium (Pseudoscorpiones: Olpiidae) from St. Eustatius (Statia), St. Martin (Sint Maarten) and Anguilla (Lesser Antilles, Leeward group). Zoologische Mededelingen 76: 451-472.

TOOREN, D. 2011. New records of olpiid pseudoscorpions (Pseudoscorpiones: Olpiidae) from the Caribbean area and Surinam, with descriptions of four new species of the genera Pachyolpium Beier, Tricholpium gen. nov. and Heterohorus gen. nov. Zoologische Mededelingen Leiden 85 (8) 29. iv: 169-194.

VACHON, M. 1951. Les Pseudoscorpions de Madagascar. I. Remarques sur la famille des Chernetidae J.C. Chamberlin, 1931, a propos de la description d'une nouvelle espece: Metagoniochernes milloti. Mémoires de l'Institut Scientifique de Madagascar 5: 159-172.

WEYGOLDT, P. 1969. The biology of pseudoscorpions. Harvard Books in Biology. 6 ed. German edition. Cambridge, Massachusetts. 